\title{
Contribution des hôpitaux de jour en psychiatrie dans le champ contemporain de la santé mentale au Québec
}

\author{
Contribuição dos hospitais-dia de psiquiatria \\ no campo da saúde mental em Quebec
}

Marie-Laurence Poirel ${ }^{1}$

Emmanuelle Khoury ${ }^{1}$

Lourdes Rodriguez $^{1}$

Nadine Larivière ${ }^{2}$

Catherine Riendeau-Janvier ${ }^{1}$

${ }^{1}$ École de service social, Université de Montréal. Université de Montréal 6128, succursale Centre-ville. H3C 3J7 Montréal Québec Canada. marie-laurence.poirel@ umontreal.ca

${ }^{2}$ École de réadaptation, Université de Sherbrooke.
Résumé Actuellement au Québec, les hôpitaux de jour en psychiatrie sont des milieux de jour offrant un programme thérapeutique intensif de courte durée, principalement dans un contexte d'activités de groupe. Alors que les hôpitaux de jour sont des acteurs qui font partie intégrante du système de soins en santé mentale depuis plusieurs décennies, la question de leur rôle et de leur contribution spécifique dans le champ des approches et des pratiques de traitement et pour les personnes qu'ils rejoignent est rarement posée. Cet article s'articule autour de l'hypothèse selon laquelle les repères et les cadres actuellement dominants dans le champ de la psychiatrie et de la santé mentale permettent mal de dégager et de prendre en compte certains des paramètres dont il faut tenir compte pour parvenir à préciser et à situer le rôle et la contribution des hôpitaux de jour, dans le système de soin comme pour les personnes auxquelles ces milieux de pratique s'adressent. À partir des résultats préliminaires d'une étude exploratoire poursuivie dans quatre hôpitaux de jour où différents acteurs ont été rencontrés lors d'entrevues (personnes utilisatrices, intervenants, gestionnaires), cet article propose de dégager quelques pistes d'analyse et de réflexion.

Mots clés Psychiatrie, Traitement, Hôpital de jour, Thérapie de groupe, Pratiques fondées sur des données probantes, Recherche qualitative
Resumo Em Quebec, atualmente, os hospitaisdia em psiquiatria oferecem um programa terapêutico intensivo de curta duração, focado em atividades de grupo. Esses hospitais-dia são parte integrante do sistema de saúde em psiquiatria e saúde mental depois de várias décadas, entretanto o seu papel em relação às suas práticas com a inclusão daqueles que seguiram esse tratamento é raramente colocado. Nessa perspectiva, este artigo se articula em torno da hipótese de que os valores de referencia, hoje dominantes no campo da psiquiatria e saúde mental, não permitem identificar e levar em conta certos parâmetros necessários para se precisar e situar o papel e a contribuição específica dos hospitais-dia no sistema de saúde mental e para as pessoas às quais se destinam. $\bar{A}$ partir de resultados preliminares de um estudo exploratório realizado em quatro hospitais-dia em psiquiatria, com entrevistas individuais (pessoas usuárias, trabalhadores e gestores), se propõe identificar algumas pistas de análise e reflexão. Palavras-chave Psiquiatria, Tratamento, Hospital-dia, Terapia de grupo, Práticas baseadas em evidencias, Pesquisa qualitativa 


\section{Introduction}

Dans la forme que l'on retrouve actuellement au Québec, les hôpitaux de jour en psychiatrie sont des milieux de traitement externes offrant un programme thérapeutique intensif de courte durée, principalement dans un contexte d'activités de groupe. Alors que les hôpitaux de jour sont des acteurs qui font partie intégrante du système de soins en psychiatrie et en santé mentale au Québec depuis plusieurs décennies, la question de leur rôle et de leur contribution spécifique est rarement posée. Ce constat et les questions qu'il soulève se trouvent à l'origine de cet article.

Le propos de cet article est double, socio-historique et clinique. Il s'intéresse à la manière dont le contexte social et culturel infléchit le regard ou l'absence de regard qui est porté sur les pratiques et donc la légitimité qui est accordée à certaines d'entre elles et moins à d'autres. Il fait l'hypothèse que les repères actuellement dominants dans le champ de la santé mentale au Québec permettent mal de prendre en compte certains des paramètres dont il faut tenir compte pour parvenir à préciser le rôle et la contribution spécifique des hôpitaux de jour, dans le système de soin en santé mentale comme pour les personnes auxquelles ces milieux de pratique s'adressent. C'est ainsi en résonance avec une lecture du contexte plus large des pratiques les plus reconnues aujourd'hui dans le champ de la santé mentale au Québec et des conditions de cette reconnaissance que les questions de la contribution spécifique des hôpitaux de jour et des conditions d'une prise en compte de cette spécificité sont explorées. À cet égard, cet article propose, à partir des résultats préliminaires d'une étude exploratoire poursuivie dans quatre hôpitaux de jour en psychiatrie, de dégager quelques pistes d'analyse et de réflexion.

\section{De l'historicité des approches et des pratiques en psychiatrie et en santé mentale}

Dans un ouvrage dont les hypothèses restent actuelles, A. Leighton a montré comment l'histoire des approches et des pratiques en psychiatrie et en santé mentale en Occident apparaît marquée par une succession de promesses suivies de désillusions ${ }^{1}$. Des approches et des pratiques réputées prometteuses à une certaine époque se verront ainsi éclipsées par d'autres qui, pour plusieurs, finiront à un moment ou à un autre par connaître un sort similaire. Leighton rappelle comment, d'une manière fondamentale, cette situation viendrait exprimer et refléter la résistance des problèmes de santé mentale graves face aux diverses réponses qui leur sont apportées, la complexité de ces problèmes et la part d'incertitude qui les traverse.

Par ailleurs, Leighton et d'autres auteurs ont montré aussi comment les pratiques et les approches en santé mentale s'avèrent fortement marquées par le contexte plus large dans lequel elles viennent s'inscrire ${ }^{1,2}$, et portent l'empreinte des conceptions et des valeurs dominantes d'une époque et d'une société comme de facteurs matériels et de circonstances propres à un temps et à un espace particulier. En fonction de circonstances internes mais aussi extérieures au champ de la psychiatrie et de la santé mentale ou qui le débordent, certaines orientations dans les approches et les pratiques se verraient privilégiées et légitimées, et d'autres mises à l'écart ou marginalisées, sans que leur rôle et contribution aient été toujours bien éprouvés. Ce type de lecture invite à la prudence devant les "évidences" de pertinence et d'efficacité qui, à une époque ou à une autre, sont attribuées à certaines approches et pratiques et aux fondements sur lesquels celles-ci reposent, au détriment d'approches qui s'appuient sur des fondements différents.

Poser ce type de regard sur le champ contemporain de la santé mentale peut permettre de requestionner et peut-être réévaluer la signification de certaines orientations et, de manière spécifique, le rôle et la contribution d'approches et pratiques de traitement qui semblent affectées par un déficit de visibilité et de légitimité.

\section{Le champ contemporain des approches et des pratiques de traitement en psychiatrie et en santé mentale au Québec: standardisation des approches et resserrement des enjeux}

Dans le contexte québécois actuel, le champ des approches et des pratiques en santé mentale apparaît marqué par une tendance à l'uniformisation et à la standardisation. Dans le domaine plus particulier du traitement, on a assisté au cours des dernières décennies à une tendance allant dans le sens d'une simplification et d'un resserrement des enjeux, qui s'accompagne et s'appuie sur une conception de moins en moins dynamique des problèmes de santé mentale graves. Ce resserrement des enjeux touche tant les finalités et les cibles du traitement, qui s'adressent d'abord aux symptômes manifestes dans les troubles mentaux, que les moyens qui sont mis en œuvre, qui visent 
d'abord les symptômes ${ }^{3}$. Au sein du champ de la santé mentale mais aussi à l'extérieur de ce champ, différents facteurs et forces semblent avoir participé à l'émergence et à l'expansion de cette double tendance à la standardisation des approches et au resserrement des enjeux du traitement. Ainsi, à l'extérieur du champ de la santé mentale, des phénomènes d'ordre structurel et d'ordre socio-culturel pourraient avoir contribué à l'évolution qu'a connue le champ de la santé mentale dans les dernières décennies au Québec et en Amérique du Nord. On peut penser aux politiques de compression dans les dépenses publiques dans un contexte de crise de l'État social qui ont favorisé la recherche d'efficacité rapide et d'efficience dans les services publics. Dans un registre complètement différent, l'effritement des repères collectifs qui avaient contribué à façonner le vivre ensemble, le rapport à soi et le sens de la présence au monde dans les époques antérieures a participé à la diffusion d'une vision du monde plus pragmatique où des questions comme celles des dimensions tragiques de la condition humaine, de la souffrance et du sens, auxquelles nous renvoient les problèmes de santé mentale graves, semblent de plus en plus évacuées ${ }^{4}$.

À l'intérieur du champ de la psychiatrie et de la santé mentale au Québec et en Amérique du Nord, la position dominante du courant biomédical, auquel la large diffusion des dernières versions de l'outil Diagnostic and Statistical Manual of Mental Disorders participe étroitement, a fortement contribué à circonscrire le traitement reconnu, légitime, au traitement psychotrope $e^{5,6}$. En tension mais aussi par certains égards en complémentarité avec le courant biomédical, certains chercheurs et cliniciens du courant du recovery, fortement imprégnés de la philosophie pragmatique de la réadaptation psychosociale, ont eu tendance à mettre davantage l'accent, dans le traitement, sur la réduction des symptômes qui hypothèquent l'intégration sociale ${ }^{7}$. Le courant biomédical et certains acteurs venant du courant du recovery semblent, chacun à leur manière, avoir contribué à un resserrement des enjeux du traitement autour d'une lecture principalement biomédicale des troubles mentaux, et à une standardisation des approches et des pratiques dans le domaine du traitement ${ }^{8,9}$.

Cette position dominante des perspectives biomédicales dans la psychiatrie québécoise et nordaméricaine contemporaine s'est vue renforcée par un troisième courant, la Nouvelle gestion publique. Sous la pression de forces sociales et socioéconomiques plus larges, la Nouvelle gestion pu- blique a pris un important ascendant dans l'organisation des soins et des services dans le domaine de la santé mentale comme plus largement dans les services sociaux et de santé, particulièrement dans le secteur institutionne ${ }^{10-12}$. Ce courant exerce des pressions importantes sur les orientations qui sont prises dont les répercussions se font sentir sur le terrain. Ainsi, des critères d'efficience tendent à dicter de manière prioritaire les orientations en matière de santé mentale. Les services et les pratiques sont d'abord évalués à partir de critères quantitatifs, objectivables (diminution des symptômes, réduction du recours à l'hospitalisation, amélioration du fonctionnement social évalué à partir de critères pré-établis). On tend à favoriser l'application de modèles de pratique qui auraient fait leurs preuves en ce qu' ils seraient fondés sur des données probantes, validées à partir de critères ciblés et objectifs (Evidences Based Practices). Dans ce contexte, certains modèles de pratique mobilisent de plus en plus l'attention des chercheurs et des gestionnaires. C'est notamment le cas des pratiques de suivi individualisé dans la communauté tel l'Assertive Community Treatment notamment reconnu pour son impact sur la réduction des taux d'hospitalisation et dont l'empreinte biomédicale a été soulignée ${ }^{13}$. La large légitimité accordée aux pratiques fondées sur les données probantes exerce une pression sur l'organisation des services et sur les pratiques en santé mentale, une pression qui, fondamentalement, va dans le sens de la standardisation. Cette pression a notamment pour effet pervers de favoriser la reproduction de pratiques réputées exemplaires, l'uniformisation plus que la diversité des approches et des pratiques. Dans ce contexte, on a l'impression qu'un étau se resserre sur des modèles de pratique qui reposent sur d'autres fondements, dont l'étude et l'évaluation exigent des instruments plus complexes et plus subtils et dont la mise en place et le développement n'ont pas correspondu à l'application de modèles pré-existants, validés par des données probantes. Ce contexte a aussi pour contrepartie un appauvrissement de la réflexion clinique.

Un souci de résultats observables et objectivables marque ainsi le champ contemporain des approches et des pratiques en santé mentale en Amérique du Nord, particulièrement dans le traitement face aux problèmes importants de santé mentale. En même temps, on assiste au renforcement de courants de pensée qui tentent de surmonter l'incertitude face aux problèmes de santé mentale graves ${ }^{12}$. Il y a quelques années, E. Corin a montré comment la quête d'évidences et de re- 
pères "sûrs" face aux problèmes de santé mentale graves prend place dans un contexte socio-culturel plus large de déstabilisation par rapport aux repères qui avaient guidé les époques antérieures et comment un tel contexte tend à s'accompagner de la recherche de nouvelles certitudes fondées entre autres sur le "roc" du biologique ${ }^{2}$.

\section{De la résistance de certaines approches et pratiques}

Au Québec et plus largement en Occident, des voix se sont élevées pour évoquer les limites des approches dominantes en psychiatrie et en santé mentale dans leur quête d'évidences dans un champ aussi complexe et incertain que celui des troubles mentaux graves ${ }^{8,14-17}$. Cette reconnaissance de complexité et d'incertitude n'a pas nécessairement pour contrepartie un constat d'impuissance et de désillusion ${ }^{14,18}$. Elle s'accompagne beaucoup plus souvent d'un pari sur des avancées prudentes, sensibles à la pluralité et à la complexité des situations et des expériences. Elle engage à la modestie et à l'humilité, suscite la créativité. Elle se conjugue avec un appel à la coexistence et au développement de différentes réponses. Dans cette perspective, certains auteurs ont rappelé l'importance d'une organisation des services qui continue à s'articuler de manière fondamentale sur des critères et des paramètres d'ordre clinique et thérapeutique, insistant en particulier sur la nécessité de continuer à poser et à penser la question des implications thérapeutiques de différents dispositifs de traitement ${ }^{19,20}$. Entre autres, la nécessité pour un système de soins en santé mentale de réussir à préserver et à développer des dispositifs de traitement suffisamment intensifs et complets qui soient établis à partir d'un lieu en tant que tel, comme c'est notamment le cas des hôpitaux de jour, a été relevée.

Concernant plus particulièrement le Québec, le constat de l'importance de l'accessibilité à une diversité d'approches d'intervention pour répondre à la diversité des situations vécues par les personnes qui vivent avec des problèmes de santé mentale est identifiée dans les documents politiques des dernières années en matière de santé mentale ${ }^{21}$. Le discours se révèle toutefois ambigu puisque l'on en appelle aussi au renforcement de certaines pratiques, réputées plus efficaces, fondées sur des données probantes, en identifiant en particulier les pratiques de suivi individualisé dans la communauté ${ }^{21}$. De manière concrète, ces pratiques se voient promues et privilégiées dans l'organisation des services en santé mentale ${ }^{22}$. Par con- tre, d'autres modèles de pratique apparaissent frappés d'invisibilité dans les discours politiques québécois des dernières années. C'est le cas des hôpitaux de jour en psychiatrie. En effet, après avoir connu une brève période de popularité et d'expansion rapide à la fin des années 1990 en tant qu'alternative à l'hospitalisation ou comme transition post-hospitalisation ${ }^{23,24}$, les hôpitaux de jour ne sont ni dans la ligne de mire des orientations publiques en matière de santé mentale ni des travaux de recherche sur les meilleures pratiques. Leur maintien semble davantage fonction de choix locaux propres à certains établissements et de dynamiques et équilibres internes. Dans les dernières années, on a par ailleurs assisté à la fermeture de quelques hôpitaux de jour en psychiatrie. Le contexte particulier de ces fermetures mériterait d'être étudié. Mais la question qui nous occupe ici est ailleurs: sur quelles bases et à partir de quels repères et critères poser et explorer la question de la contribution des hôpitaux de jour dans le champ contemporain du traitement en santé mentale au Québec et pour les personnes qui fréquentent ces milieux de pratique?

De façon plus large, pour tenter de résister à l'invisibilité et à la marginalisation, deux voies semblent s'ouvrir pour les approches et les pratiques restées jusqu'ici largement à l'écart de l'attention et de la reconnaissance des pratiques fondées sur des données probantes: tenter de se faire évaluer et peut-être reconnaître à partir du même cadre ou s'attacher à faire ressortir la pertinence et l'importance d'autres types de repères et de critères. Ce qui peut revenir aussi à relever les limites des pratiques fondées sur des données probantes dans le champ de la santé mentale et la pression parfois indue sous forme de rouleau compresseur que leur sur-investissement symbolique et concret fait peser sur ce champ. Notre hypothèse est précisément que l'étude de la contribution des hôpitaux de jour dans le champ contemporain de la santé mentale demande de faire appel à d'autres types de repères et de critères que ceux qui sont mobilisés dans la perspective des pratiques fondées sur des données probantes.

Au Québec comme plus largement en Amérique du Nord, on constate dans la littérature un manque relatif d'études portant sur l'hôpital de jour en psychiatrie comme approche de traitement à part entière, mis à part un certain nombre d'études réalisées par des représentants des milieux de pratique concernés ${ }^{24-27}$. Ce manque relatif a notamment été mis en relation avec la tendance, de plus en plus présente dans la recherche en psychiatrie, à circonscrire le cadre et les 
cibles du traitement à une action sur la symptomatologie. ${ }^{26}$ Or, les études disponibles sur le traitement en hôpital de jour mettent précisément en évidence une approche de traitement qui déborde l'action sur la symptomatologie, qui implique un abord plus large de la personne touchée par des troubles mentaux à travers la mobilisation d'une diversité de moyens thérapeutiques et de dispositifs ${ }^{26,28}$. Au Québec, une étude qualitative réalisée dans différentes équipes cliniques d'un hôpital de jour en psychiatrie explorant le point de vue de personnes utilisatrices arrive au même type de conclusions ${ }^{29}$.

Par certains aspects, les résultats des études monographiques et qualitatives réalisées dans des hôpitaux de jour en psychiatrie rejoignent les résultats d'autres recherches de nature qualitative poursuivies dans d'autres types de milieux de pratique dont les dispositifs présentent certains rapprochements avec les hôpitaux de jour. Ainsi, une recherche récente menée au Québec dans des milieux de pratique non institutionnels qui s'identifient comme des ressources alternatives de traitement en santé mentale a mis en évidence l'importance et la contribution, d'un point de vue thérapeutique, d'une approche de traitement qui se développe à partir d'un lieu en tant que tel et qui sollicite une pluralité de moyens et d'activités thérapeutiques ${ }^{8,30}$. Cette recherche a notamment montré la contribution de dimensions qui tiennent à l'atmosphère qui prévaut dans un milieu de pratique et la place de la vie communautaire et du groupe au cœur des dispositifs. La recherche a permis de dégager un effet d'ensemble des dispositifs. En même temps, elle a fait ressortir la difficulté voire l'impossibilité d'isoler les différents "ingrédients" thérapeutiques dans la compréhension des processus à l'œuvre et l'importance de les appréhender dans une perspective dynamique. Par ailleurs, cette recherche poursuivie dans des ressources alternatives de traitement a mis en évidence la spécificité de la philosophie de traitement dans laquelle s'inscrivent ces milieux de pratique. Ces derniers, rejoignant l'esprit de certaines traditions de la psychiatrie européenne $e^{16,31}$, mettent en effet de l'avant une conception du traitement dans laquelle l'attention privilégiée concerne la subjectivité de la personne souffrante ${ }^{3}$.

La recherche dans des ressources alternatives de traitement a impliqué le développement d'instruments et d'une stratégie qualitative de cueillette de données et d'analyse qui permettent d'explorer en profondeur et dans une perspective dynamique un milieu de pratique à partir du point de vue des principaux acteurs impliqués et, donc de faire ressortir les dimensions des pratiques et des dis- positifs qui, de l'intérieur, apparaissent pertinents d'un point de vue thérapeutique ${ }^{8}$. Une série de rubriques d'analyse ont été dégagées qui relèvent de l'atmosphère, de la philosophie, des principes thérapeutiques et des dispositifs. Les instruments développés dans cette recherche présentent plusieurs points communs avec des instruments élaborés par R. Moos pour l'étude de milieux de pratique en psychiatrie et dans le domaine socio-sanitaire qui prennent en compte à la fois les dispositifs, l'atmosphère et la philosophie de pratique ${ }^{32}$. Dans les deux cas, les instruments et les stratégies utilisés permettent de poser et d'explorer la question fondamentale des implications thérapeutiques d'un programme de traitement considéré dans son ensemble, à partir d'une diversité de dimensions. Dans une étude exploratoire menée dans des hôpitaux de jour en psychiatrie qui avait pour but d'explorer et de dégager les principaux paramètres permettant de préciser la spécificité de ces milieux de pratique, nous nous sommes inspirées de la stratégie de cueillette des données et d'analyse mise au point dans la recherche ayant impliqué des ressources alternatives de traitement.

\section{Pour repenser le rôle et la contribution des hôpitaux de jour en psychiatrie: une étude qualitative dans quatre hôpitaux de jour québécois}

L'étude exploratoire visait ainsi à dégager les principaux paramètres des programmes d'hôpital de jour en psychiatrie pertinents d'un point de vue clinique et thérapeutique, et ce de la perspective des acteurs directement impliqués, des usagers, des intervenants (infirmières, travailleuses sociales, psychologues, éducatrices spécialisées) et des gestionnaires (cadres administratifs dans certains cas, psychiatres dans d'autres). Quatre hôpitaux de jour en psychiatrie de la région de Montréal ont participé à cette démarche de recherche qualitative. Ces quatre hôpitaux de jour couvrent ensemble un large spectre de diagnostics psychiatriques: troubles psychotiques, troubles de la personnalité, troubles de l'humeur et troubles anxieux. Dans chaque hôpital de jour, des entretiens semi-dirigés ont été réalisés avec deux personnes qui avaient complété le programme, deux intervenants et un gestionnaire, pour un total de vingt entrevues d'une durée d'une heure à deux heures trente. Les entrevues ont été enregistrées et retranscrites de façon systématique.

Pour tenter de dégager certains traits caractéristiques des hôpitaux de jour en psychiatrie et, à partir de là, de mieux situer la spécificité de leur 
contribution, il s'agissait ainsi non de partir de critères pré-établis mais plutôt de ce qu'en disent les acteurs les plus directement impliqués. Nous présentons ici quelques pistes d'analyse et de réflexion à partir d'une analyse préliminaire des entrevues avec ces différents acteurs en fonction de paramètres touchant en particulier à la philosophie de pratique et aux dispositifs.

De manière générale, l'étude a permis de dégager deux types d'hôpital de jour. Un premier type implique une spécialisation en fonction des diagnostics psychiatriques. La structure du programme y apparaît clairement établie et évite les espaces et les moments informels. Le travail thérapeutique est circonscrit aux activités structurées. Les relations entre usagers de même qu'entre intervenants et usagers y apparaissent plus balisées. On pourrait dire que le cadre apparait ici plus investi que ce qui touche à l'atmosphère. Le deuxième type accepte ou s'accommode d'une plus grande diversité dans les problèmes de santé mentale vécus par les personnes. Une flexibilité plus grande caractérise de façon générale ici les hôpitaux de jour. Tout en proposant un programme structuré d'activités, ces hôpitaux de jour ont mis en place des espaces informels dont on reconnait l'importance sinon la pertinence sur un plan thérapeutique. Une plus grande proximité semble présente dans les relations entre usagers et intervenants. L'atmosphère de l'hôpital de jour est décrite comme un paramètre important des pratiques.

Par ailleurs, au-delà de ces différences qui pourront faire l'objet d'une analyse ultérieure plus approfondie, l'analyse transversale préliminaire des entrevues des différents acteurs met en évidence de manière frappante que les hôpitaux de jour impliqués dans notre étude sont, de l'intérieur, traversés et travaillés par des tensions. Or, il nous est apparu que ces tensions devaient être sérieusement prises en compte dans l'analyse et la réflexion sur la contribution de ces milieux de pratique dans le champ contemporain du traitement en santé mentale. À cet égard, nous soutenons que les tensions qui semblent travailler de l'intérieur les hôpitaux de jour qui ont participé à cette étude apparaissent autant sinon davantage le signe de leur vitalité et de leurs potentialités d'un point de vue clinique et thérapeutique que le révélateur des incertitudes et des limites de ces milieux de pratique. Nous allons voir que les principales tensions se dessinent dans un écart entre la philosophie, les buts et les objectifs qui sont affichés, les approches qui sont privilégiées, d'une part, et, d'autre part, les potentialités du dispositif qui est déployé au sein de l'hôpital de jour.

\subsection{Un projet modeste}

Le projet thérapeutique des hôpitaux de jour impliqués dans notre étude apparaît somme toute assez modeste. On est loin d'un discours critique appelant à un renouvellement de la compréhension des problèmes de santé mentale à partir d'une prise en compte de la subjectivité et au développement de réponses qui soient à l'écoute d'une telle compréhension. Le projet de ces hôpitaux de jour n'a à première vue rien de subversif par rapport aux orientations aujourd'hui dominantes en psychiatrie et en santé mentale au Québec. Par les principales cibles qui sont visées dans le travail thérapeutique, par les finalités qui sont identifiées et par les principales approches qui sont utilisées, ce projet semble même largement s'inscrire dans le mainstream de la psychiatrie nordaméricaine contemporaine.

L'un des principaux traits des hôpitaux de jour en psychiatrie du Québec est qu'il s'agit de milieux de pratique qui s'inscrivent dans un relatif court terme (correspondant en moyenne à huit semaines). Les différents acteurs rencontrés reviennent beaucoup sur ce trait et en mettent en évidence les implications. Ce trait façonne de manière importante le projet poursuivi et les approches qui sont privilégiées. Ainsi, les différents acteurs mettent en évidence la visée d'abord pragmatique des hôpitaux de jour. Les finalités du projet porté par l'hôpital de jour sont évoquées en termes de soutien à une réappropriation de la vie quotidienne et à un retour à la vie dans la collectivité après une période de déstabilisation. Les cibles qui sont principalement identifiées concernent les symptômes, les difficultés fonctionnelles dans la vie quotidienne et la vie sociale. Comme le remarquent certains participants à l'étude, l'hôpital de jour travaille fondamentalement dans l'ici et maintenant. On y donne une grande place à une démarche par objectif. Les méthodes psycho-éducatives et les approches cognitivo-comportementales semblent souvent s'être imposées comme approches et méthodes privilégiées. L'éducation par rapport aux symptômes et aux diagnostics est partie intégrante du programme d'activités, sans que le travail psycho-éducatif épuise par ailleurs le sens des activités. Nous y reviendrons. La médication compte aussi parmi les moyens sur lesquels les hôpitaux de jour s'appuient.

Si les témoignages recueillis font ressortir la relative modestie du projet thérapeutique de l'hôpital de jour, ils mettent aussi en évidence le potentiel du dispositif qui caractérise les hôpitaux de jour impliqués dans notre étude. Pour mieux 
saisir certains aspects de la spécificité de la contribution des hôpitaux de jour, il semble ainsi que nous devions nous tourner vers leur dispositif.

\subsection{Les potentialités du dispositif}

De manière générale, les hôpitaux de jour qui ont participé à notre étude semblent se définir davantage par rapport à un certain dispositif que par rapport à une philosophie de pratique en tant que telle. - Ils ne s'éloignent en fait guère en cela d'autres types de modèles de pratique qui existent au Québec aujourd'hui dans les services institutionnels de psychiatrie et de santé mentale. Par ailleurs, par sa diversité et sa vitalité, le dispositif qui est mis en place dans l'hôpital de jour donne une couleur et une direction spécifique à la pratique. À travers lui, l'hôpital de jour pourrait, dans une certaine mesure, d'un point de vue clinique et thérapeutique, être entraîné au-delà du projet modeste qu'il affiche. Deux traits du dispositif des hôpitaux de jour se dégagent en particulier des entrevues des différents acteurs: l'intensité du programme et le rôle central du groupe dans le programme. L'un et l'autre font ressortir l'existence et la mobilisation d'une dimension dynamique dans le programme et les processus de traitement à l'œuvre dans ces milieux de pratique.

\subsubsection{L'intensité}

Dans le système de soins institutionnel en santé mentale au Québec, les hôpitaux de jour proposent une intensité de pratique qui n'a guère d'équivalent. Pendant huit semaines, les personnes engagées dans ce type de programme fréquentent de manière quotidienne l'hôpital de jour où elles sont appelées à participer à un programme structuré d'activités psycho-éducatives et thérapeutiques. À cet égard, les entrevues avec différents acteurs se font écho pour évoquer une intensité différente de ce qui existe ailleurs dans le système public, qui amènerait la personne dans une mobilisation particulièrement intense face aux difficultés qu'elle éprouve.

Par ailleurs, les témoignages montrent aussi que l'hôpital de jour intervient souvent à une étape particulière dans une trajectoire personnelle, à un moment charnière dans un parcours de vie et de problèmes de santé mentale. Ce moment charnière est identifié dans plusieurs cas comme un moment de crise ou une situation critique, aiguë, face à laquelle la personne et les intervenants qui l'accompagnent s'entendent pour reconnaître que les choses ne peuvent plus continuer et doivent changer. L'hôpital de jour en psychiatrie semble ainsi beaucoup miser sur une large mobilisation face à la crise, à laquelle est conviée la personne mais qui concerne aussi l'ensemble d'un programme et le personnel qui y travaille. À cet égard, les témoignages recueillis suggèrent que, pour répondre adéquatement à la crise, l'intensité de pratique particulière, par sa durée et sa qualité, qui caractérise l'hôpital de jour pourrait être particulièrement indiquée.

Une compréhension de la crise qui déborde une interprétation biomédicale se dégage ainsi de plusieurs témoignages qui mettent en évidence que la crise et le potentiel de la crise sont pris au sérieux et font l'objet d'un travail thérapeutique dans les hôpitaux de jour en psychiatrie. Une dimension dynamique est en ce sens introduite dans l'approche et le programme de traitement. Elle vient témoigner de la reconnaissance, certes plus souvent implicite qu'explicite, qu'une réponse unique comme la réponse médicamenteuse ne suffit pas face à la crise dès lors que celle-ci est reconnue comme moment charnière dans une trajectoire personnelle. Cette compréhension sous-jacente de la crise qui s'accompagne de la mobilisation de stratégies diversifiées semble amener ces milieux de pratique, peut-être comme malgré eux, à repousser les limites d'une approche strictement biomédicale.

\subsubsection{Le groupe}

Le groupe est au cœur des dispositifs de l'hôpital de jour. L'importance du groupe est mise en évidence dans l'ensemble des témoignages des différents acteurs. Les diverses activités mises en place le sont principalement dans un contexte de groupe. Différentes facettes sont évoquées. Le groupe est parfois utilisé de manière plus statique sous la forme d'enseignements dispensés aux participants. Une partie du travail de groupe répond ainsi à des objectifs psycho-éducatifs. D’autres activités de groupe visent plus spécifiquement des thématiques comme l'estime de soi et les difficultés relationnelles. Certaines activités réalisées en groupe sont conçues pour travailler la mobilisation du plaisir; cette dimension étant désignée comme importante dans les hôpitaux de jour en psychiatrie.

Les témoignages montrent aussi que, dans plusieurs circonstances et à travers différentes activités, le groupe est mobilisé dans une perspective directement thérapeutique où les participants sont appelés à jouer un rôle actif et à interagir entre eux à partir de balises beaucoup plus fluides, mettant en évidence que certaines activités sollicitent directement la dynamique et le travail de groupe en 
tant que tel. Plusieurs acteurs parlent ici de thérapie de groupe, évoquant la mobilisation du groupe dans un travail thérapeutique.

En misant sur le groupe comme entité dynamique, sur ce qui peut se jouer et advenir dans et par le travail de groupe, en acceptant ainsi l'irruption de facteurs qui ne peuvent ultimement être contrôlés, en laissant place à une dimension d'imprévisibilité, le dispositif de l'hôpital de jour semble impliquer la reconnaissance implicite d'une part nécessaire d'incertitude dans tout travail thérapeutique dans le domaine de la psychiatrie et de la santé mentale. Avec ce type de recours au groupe, c'est un facteur foncièrement dynamique qui est introduit dans les pratiques.

\section{En guise de conclusion: un écart bénéfique}

C'est à travers la mise en évidence, à l'intérieur même de l'hôpital de jour en psychiatrie, de certaines tensions que nous avons commencé à explorer la question de la spécificité de la contribution de ces milieux de pratique dans le champ du traitement en santé mentale au Québec aujourd'hui. Dans un champ marqué par une forte tendance à la standardisation, à l'uniformisation et au resserrement des enjeux, l'hôpital de jour tel qu'il se dégage de notre étude semble venir introduire, avec prudence, la possibilité d'un écart, peut-être aussi d'une ouverture. En effet, de manière plus implicite qu'explicite, l'hôpital de jour en psychiatrie, à travers son dispositif impliquant la mobilisation d'un ensemble de pratiques diversifiées dans lequel le groupe s'avère central, nous a semblé mettre en œuvre une pratique de traitement qui élargit le cadre de l'approche biomédicale.

Il y a plusieurs années, dans une réflexion qui reste toujours très actuelle, le psychanalyste R.C. Racamier s'est intéressé à la place donnée au traitement biomédical et en particulier à la médication dans un milieu de traitement en psychiatrie $^{33}$. Il a distingué à cet égard deux principaux types de milieux. D'un côté, un type de milieu de pratique qui donne un rôle central aux médicaments psychiatriques lesquels ordonnent l'ensemble de la pratique et les relations entre soignants et soignés de même qu'entre soignants. D'un autre côté, un type de milieu de pratique où la médication occupe une place plus secondaire et instrumentale, où elle ne résume ni le traitement ni les relations. Dans le premier cas, on peut voir à l'œuvre une pratique de traitement plus mécanique où une réponse unique et ciblée, la médication, est apportée à un problème ciblé, un ou des symptômes. Dans le deuxième cas, on a affaire à une pratique et à un processus beaucoup plus complexes, diversifiés et dynamiques. La figure de l'hôpital de jour tel qu'il ressort de notre étude se rapproche davantage de ce deuxième type de milieu de pratique.

En effet, en mettant à contribution dans le traitement face aux problèmes de santé mentale graves des dimensions dynamiques telle la reconnaissance de la crise comme moment charnière dans une trajectoire et en donnant au travail du groupe un rôle central, l'hôpital de jour élargit le cadre des paramètres à considérer dans le traitement. Dans un contexte où le dispositif implique un ensemble de pratiques diversifiées, la médication apparaît comme un ingrédient parmi d'autres. C'est ainsi une conception du traitement qui tout à la fois reconnaît et limite la place et le rôle du traitement biomédical qui se dégage de notre étude. Sans que ce soit directement exprimé et malgré un projet thérapeutique qui reste assez circonscrit, cette orientation du dispositif de l'hôpital de jour amène ainsi un certain déplacement par rapport à l'approche biomédicale.

Un tel déplacement se révèle par ailleurs prudent et partiel. L'hôpital de jour tel qu'il se découvre à travers notre étude apparaît aussi l'expression d'une compréhension aujourd'hui dominante du traitement en santé mentale qui va dans le sens du pragmatisme, et qui laisse de côté tout un pan de la réflexion et de l'expérience du traitement face aux problèmes de santé mentale graves. L'hôpital de jour en psychiatrie tel qu'il ressort de notre étude témoigne d'une compréhension plus horizontale que verticale du traitement par laquelle l'expérience subjective de la souffrance au cœur des problèmes de santé mentale graves se verrait en tant que telle prise en compte.

\section{Contributions}

ML Poirel a assuré la direction scientifique de la recherche à ses différentes étapes et rédigé la première version de l'article ainsi que sa version finale. E Khoury a contribué de manière significative à la réalisation de la recherche sur le plan méthodologique. L Rodriguez et $\mathrm{N}$ Larivière ont contribué à la direction scientifique de la recherche et à la version finale du texte. C Riendeau-Janvier a participé à la cueillette et à l'analyse des données. 


\section{Références}

1. Leighton AH. Caring for mentally ill people. Psychological and social barriers in historical context. Cambridge: Cambridge University Press; 1982.

2. Corin E. Impasses et défis des psychothérapies psychanalytiques dans le monde contemporain PRISME 1996; 6(2-3):333-349.

3. Poirel ML, Corin E. Revisiter la notion de traitement en santé mentale et en psychiatrie du point de vue de personnes usagères et d'intervenants de ressources alternatives en santé mentale: la question de la subjectivité. Santé mentale au Québec 2011, 36(1):115-130

4. Fédida P. Des bienfaits de la dépression. Éloge de la psychothérapie. Paris: Éditions Odile Jacob; 2001.

5. Dufort F, Poirel ML, Scheibler P, Thoer C. À propos de quelques incursions de la psychologie dans la chaîne du médicament: Le cas des médicaments psychotropes. In: Lévy J, Garnier C. La chaîne des médicaments. Québec: Presses de l'Université du Québec; 2007. p. 367-396.

6. Rodriguez L, Corin E, Poirel ML. Le point de vue des utilisateurs sur l'emploi de la médication en psychiatrie: une voix ignorée. Revue québécoise de psychologie 2001; 12(2):201-223.

7. Corin E. Se rétablir après une crise psychotique: ouvrir une voie? Retrouver sa voix? Santé Mentale au Québec 2002; 27(1):65-82.

8. Corin E, Poirel ML, Rodriguez L. Le mouvement de l'être. Paramètres pour une approche alternative du traitement en santé mentale. Québec: Presses de l’Université du Québec; 2011.

9. Davidson L, Schmutte T, Dinzeot T, Andres-Hyman R. Remission and Recovery in Schizophrenia: Practitioner and Patient Perspectives. Schizophr Bull 2008; 34(1):5-8.

10. Lafortune D. Introduction: L'intervention à l'aune des données probantes. Criminologie 2009; 42(1):312.

11. Couturier Y, Carrier S. Pratiques fondées sur les données probantes en travail social: un débat émergent. Nouvelles pratiques sociales $2003 ; 16(2): 68-79$.

12. Poirel ML, Corin E. Le savoir à l'épreuve de l'incertitude dans l'intervention en santé mentale. Revue québécoise de psychologie. Soumis

13. Gélinas D. Points de repères pour différencier la gestion de cas du suivi intensif dans le milieu auprès de personnes souffrant de troubles mentaux graves. Santé mentale au Québec 1998; 13(2):17-47.

14. Sassolas M. La psychose à rebrousse-poil. Toulouse: Éditions Érès; 2009 (1997 pour la première édition).

15. Franks V. Evidence-Based Uncertainty in Mental Health Nursing. J Psychiatr Ment Health Nurs 2004; 11(1):99-105.

16. Aulagnier P. La violence de l'interprétation. Paris: PUF; 2010 (1975 pour la première édition)

17. Apollon W, Bergeron D, editeurs. Traiter la psychose. Québec: Éditions du Gifric; 1990.

18. Barazer C, Ehrenberg C. La folie perdue de vue. Esprit 1991; 9(20):29-39.
19. Bachrach LL. A Conceptual Approach to Deinstitutionalization. Hosp Community Psychiatry 1978; 29(9):573-578.

20. Sassolas M, editeurs. Malaise dans la psychiatrie. Toulouse: Éditions Érès; 2004.

21. Canada. Québec. Ministère de la Santé et des Services sociaux (MSSS). Plan d'action en santé mentale 2005-2010. La force des liens. Québec: MSSS; 2005.

22. Latimer E. Soutien d'intensité variable (SIV) et rétablissement: que nous apprennent les études expérimentales et quasi expérimentales? Santé mentale au Québec 2011; 36(1):13-34.

23. Association des hôpitaux du Québec. Portrait des hôpitaux de jour en psychiatrie du Québec. Montréal: Association des hôpitaux du Québec; 2000.

24. Boisvert D, Lecomte Y, editeurs. Hôpital de jour. Une nouvelle chance. Hôpital de jour du Centre hospitalier Pierre Janet. Santé mentale au Québec, édition spéciale; 2005.

25. Larivière N, Mélançon L, Fortier L, David P, Cournoyer J, Baril MC, Bisson J. A Qualitative Analysis of Clients' Evaluation of a Psychiatric Day Hospital. Canadian Community Ment Health J 2009; 28(1):165-179.

26. Rosie JS, Azim HFA, Piper WE, Joyce AS. Effective Psychiatric Day Treatment: Historical Lessons. Psychiatric Services 1995; 46(10):1019-1026.

27. Thibodeau J. Le Tremplin, hôpital de jour francophone. Montréal: C.H. Douglas; 1995.

28. Holloway F. Psychiatric day care: The users' perspective. Int J Soc Psychiatry 1988; 35(3):252-264.

29. Larivière N, Boyer R. Revisiter l'expérience en hôpital de jour six mois après le congé: parcours et perceptions des personnes utilisatrices. Santé mentale au Québec 2011; 36(1):75-97.

30. Poirel ML, Corin E, Rodriguez L. Traiter dans la communauté. Un regard alternatif sur des enjeux de bonnes pratiques en santé mentale. Revue canadienne de santé mentale communautaire 2012; 31(2):1-16.

31. Jaspers K. De la psychothérapie. Étude critique. Paris: PUF; 1956.

32. Moos RH. Evaluating Treatment Environments. A Social Ecological Approach. New York: Wiley-Interscience Publication; 1974.

33. Racamier PC, Diatkine R, Lebovici S, Paumelle P. Le psychanalyste sans divan. La psychanalyse et les institutions de soin psychiatrique. Paris: Payot; 1973.

Artigo apresentado em 23/03/2013

Aprovado em 21/05/2013

Versão final apresentada em 06/06/2013 